\title{
Lateral First Diagonal Branch Artery
}

National Cancer Institute

\section{Source}

National Cancer Institute. Lateral First Diagonal Branch Artery. NCI Thesaurus. Code C102307.

The lateral branch distal to a bifurcation of the first diagonal artery. 